\title{
6
}

\section{Nonresponse and attrition in a probability-based online panel for the general population}

\author{
Peter Lugtig, $^{a, c}$, Marcel Das ${ }^{b}$, and Annette Scherpenzeel ${ }^{a}$ \\ ${ }^{a}$ Utrecht University, The Netherlands \\ ${ }^{\mathrm{b}}$ CentERdata, Tilburg University, The Netherlands \\ ${ }^{\mathrm{c}}$ University of Essex, United Kingdom
}

\subsection{Introduction}

Online panel surveys have changed social and market research. Especially in applied market research, online panels are a very important tool for conducting surveys. In the early 2000s, nearly all online panels were based on self-selected samples of respondents who have access to the Internet. These self-selected panels offer quick and cheap data collection. This comes at the price of low external validity. ${ }^{1}$ Thus, self-selected panel respondents are likely to differ from the population to which the results from these respondents are meant to generalize. More importantly, the nature and size of these potential biases can never be properly assessed, because sampling theories do not apply to studies that do not rely on a random sampling scheme. This lack of external validity has continuously concerned academic researchers and people working in official statistics.

\footnotetext{
${ }^{1}$ External validity refers to how well the findings of a research study generalize to the population the study purports to represent (cf. Campbell \& Stanley, 1966; Borsboom, Mellenbergh, \& van Heerden, 2004).
}

Online Panel Research: A Data Quality Perspective, First Edition.

Edited by Mario Callegaro, Reg Baker, Jelke Bethlehem, Anja S. Göritz, Jon A. Krosnick and Paul J. Lavrakas.

(C) 2014 John Wiley \& Sons, Ltd. Published 2014 by John Wiley \& Sons, Ltd.

Companion website: www.wiley.com/go/online_panel 
Typically self-selected panels draw quota samples from a large database of self-selected respondents, and they can exclude any panel members who did not respond to earlier survey requests. Biases caused by undercoverage, nonresponse and attrition are ignored to a great extent.

To overcome the problem of self-selection, one may draw randomly from the target population and recruit a panel from this sample. When the target population also includes households without Internet access, providing selected households with access solves the coverage problem. Alternatively, those households without Internet may be interviewed using another mode (e.g., mail). This chapter discusses how problematic nonresponse and attrition are in panels where respondents are recruited offline and those without Internet access are given Internet access.

The most notable examples of probability-based online panels are those run by GfK Knowledge Networks (http://www.knowledgenetworks.com/knpanel/) and RAND (https: //mmicdata.rand.org/alp/) in the United States, and by CentERdata in the Netherlands (http: //www.centerdata.nl/en). In order to solve the undercoverage problem that not all households have access to the Internet, households in these panels are offered Internet access if they do not yet have access (Scherpenzeel \& Das, 2011). ${ }^{2}$ New initiatives modeled along the lines of CentERdata's LISS panel (see Section 6.3) have recently been started in France (ELIPSS, Étude Longitudinal par Internet Pour les Sciences Sociales; Centre for Socio-political data at Sciences Po) and Germany (GIP, German Internet Panel). The German GESIS Omnibus Panel also includes non-Internet households, but instead of providing them with equipment, these households are approached by mail.

Nonresponse and attrition are problematic in all panel surveys, especially when nonresponse rates are different for specific sub-groups of the population. From earlier studies into attrition in offline, interviewer-administered panel surveys, we know about some of the correlates of attrition. However, nonresponse and attrition may have a different nature in online self-administered panel studies than in offline panels. Because email and the Internet are the primary survey modes in online surveys, they might attract different types of respondents while repelling other groups of respondents as compared to traditional offline panel surveys. Also, over the course of the panel, because of differences in the survey process, and the absence of an interviewer, attrition may be different between online and offline panel surveys. This may lead to a decrease in statistical power, or different biases in online panel surveys than in offline panel surveys.

This chapter aims to answer the following question: What differences can we expect between the correlates of attrition in online panels and offline panels? Using an ongoing online panel dataset from the Netherlands spanning about 50 waves, we illustrate a method that can be used to study the correlates of attrition. For this dataset, we illustrate the correlates of attrition for various groups of respondents and demonstrate that each group follows a distinct attrition pattern over time. Finally, we compare the correlates of attrition with the correlates of initial nonresponse during the recruitment phase of the online panel, in order to see whether the correlates for initial nonresponse and attrition over time differ or not.

\footnotetext{
${ }^{2}$ Leenheer and Scherpenzeel (2013) find that 50\% of LISS-households who have Internet acccess, do generally not use their computer for other things than completing the questionnaires, while the other $50 \%$ become very light computer users. Further, people do not change their leisure time after being given Internet access. This implies being given Internet access does not radically change these households.
} 


\subsection{Attrition in online panels versus offline panels}

The contact mode (email) and data collection mode (Internet) make an online panel different from offline panels, which are mostly recruited and conducted through face-to-face interviewing, often complemented with mail, and telephone as secondary modes (cf. Lynn, 2009).

During the recruitment phase of an online panel study, specific groups of respondents might not be willing to complete surveys on the Internet, because they feel unable to do so. People likely to be negative towards the Internet as a survey mode are those who dislike using computers, and specifically the elderly (De Leeuw \& Hox, 2011). An earlier experiment by Olson, Smyth, and Wood (2012) has found that some people do not prefer the Internet as a survey-mode, but that almost everyone was open to a mail survey, independent of preferred survey mode. Those people who not prefer online surveys are likely to come from a specific stratum of the target population (Olson, Smyth, \& Wood, 2012). So, online panels face larger challenges in the recruitment phase than offline panel surveys. First, they have to make respondents switch from the recruitment mode (offline) to the interview mode (online). Second, some people dislike the Internet as a survey mode. For these reasons, age might be a more important correlate of initial nonresponse in online panels. Some important correlates of nonresponse in cross-sectional surveys are also likely to affect initial nonresponse in panel surveys: people in single households are less likely to be contacted, as are people in highly urbanized areas (Groves \& Couper, 1998).

Even when respondents are capable of participating in an online panel, nonresponse in any particular wave of data collection can be very high. Email invitations are used to invite respondents for new waves, and these can easily be forgotten or ignored, possibly leading to higher levels of wave nonresponse than in offline panel surveys. In offline panels, requests for survey participation are usually communicated by an interviewer, making it far less likely that requests are forgotten or ignored. Furthermore, online panels differ from offline panel surveys in the frequency of data collection. In offline panel surveys, interviews are typically conducted annually, whereas in online panels, data collection occurs far more frequently: monthly, or even weekly. Because of the high frequency of data collection in online panel surveys, there are more occasions for respondents not to respond.

These differences imply that methods to prevent nonresponse and attrition may differ between online and offline panels. In offline panels, it is important to keep contact with respondents in between waves of data collection. This is done using change-of-address cards and recording as many contact details as possible (Laurie \& Lynn, 2009). More recently, contingent monetary incentives have been offered upon participating in a wave to make sure respondents keep up at least their extrinsic motivation (Laurie \& Lynn, 2009; Lipps, 2009; see also Chapter 1 in this volume). Several offline and online panels also try to use communication strategies to keep respondents intrinsically motivated in panel participation, so as to prevent attrition. For this, they use newsletters, season/birthday greetings, and appreciation of participation cards, all of which strive to communicate the importance of participation (Laurie \& Lynn, 2009). Chapter 9 in this volume notes that these methods are not always effective, however.

Interviewers play a key role in contacting respondents in offline panels, and trying to ensure the interview is a pleasant experience for respondents. Using high caliber interviewers for those more likely to drop out (Voorpostel \& Lipps, 2011), monitoring the quality of interviewers (Schaan, 2011), and refusal avoidance training for interviewers (Kroh, 2011; 
Uhrig, 2008; Voorpostel \& Lipps, 2011), have been found to be successful in lowering attrition.

Online panel surveys do not use interviewers, but rely on email as the main mode of contact and the Internet for administration of the survey. Non-contacts may occur when respondents do not read emails, but as long as multiple email addresses and phone numbers are collected for every respondent, survey managers can usually trace and contact respondents.

Earlier studies into the correlates of attrition have all used offline panel surveys. Some of the correlates of attrition found in those studies may for that reason not apply to online panel surveys. In this chapter, we limit our analyses to covariates for which we have validation data at the population level. Of those, it has been shown that women attrite less often than men (Behr, Bellgardt, \& Rendtel, 2005; Lepkowski \& Couper, 2002). Women are thought to be more conscientious and more committed and thus miss fewer waves, although evidence for this is mixed (Uhrig, 2008). Other correlates of attrition are marital status (never being married), whether someone has moved or is planning to move (Lillard \& Panis, 1998), and the size of the household (Lipps, 2009). The fact that household composition is important may be due to persuasion by other household members to stay involved in the panel survey or also drop out. Age has been found not to be related to attrition, although the oldest old and children around the age of 18 are more at risk (Lipps, 2009).

As emails are easily ignored or forgotten, the only method that can effectively be used to prevent attrition is trying to keep respondents' motivation high. As noted above, contingent incentives are routinely offered for every wave that is completed. Because both the contact mode and the interview itself are far less personal in online than in offline panel surveys, it is very likely that attrition is a far more common phenomenon in online panel surveys, than it is in offline surveys. Moreover, because of the high frequency of data collection and greater tendency for nonresponse associated with that, it also is likely that the nature of attrition is far more varied, with respondents dropping out, and coming back to the panel survey regularly. This also has implications for the way attrition should be studied.

When investigating attrition bias in offline panels, some researchers pool all wave-on-wave attrition patterns (Nicoletti \& Peracchi, 2005; Sikkel \& Hoogendoorn, 2008; Watson \& Wooden, 2009), and simply discern two groups: the attriters and stayers. This approach ignores the possibility that attrition is different, for example, between Waves 2 and 3 and Waves 7 and 8. Moreover, it does not acknowledge or allow for individual response propensities to change with time. Another approach is to study nonresponse separately for every wave-to-wave transition (Uhrig, 2008). Apart from the fact that this requires many analyses, it is hard to deal with respondents returning to the survey, which implies that respondents can attrite multiple times. Other authors have focused only on the final state of attrition, and have limited themselves to predicting whether attrition occurs or not (Tortora, 2009), or they use duration models controlling for wave effects (e.g., Lipps, 2009). Sikkel and Hoogendoorn (2008) look at the correlates of psychological background variables with the duration of panel membership (in months) and find no relationship.

Durrant and Goldstein (2010) took a more integrative approach and looked at all possible monotonic attrition patterns in a four-wave panel study. With non-monotonic attrition, and longer panel spans, this approach is also challenging. Finally, Voorpostel (2009) and Behr et al. (2005) followed the example of Fitzgerald et al. (1998) and separate a group of attriting ("lost") respondents from returning ("ever out") respondents, thereby also allowing for non-monotonic attrition. In online panel surveys, this last approach of separating "staying," "lost," and "ever out" respondents seems promising. As online panel studies mature, different 
groups of "ever-out," and "lost" respondents are likely to appear. Thus, from a theoretical and managerial perspective, it makes sense to separate those respondents who only miss a few waves, from those who miss many. Similarly, it is also useful to separate the very fast attriters from those respondents who attrite after many waves of data collection.

Before we turn to a more technical description of how such groups can easily be separated in any set of panel response data, we will briefly describe the dataset that we are using as an example in this chapter.

\subsection{The LISS panel}

The data for our study stem from the Longitudinal Internet Studies for the Social sciences (LISS) panel, administered by CentERdata in the Netherlands. More information about the recruitment of the panel, response percentages for all waves, as well as the full questionnaires, can also be found on www.lissdata.nl or in Scherpenzeel and Das (2011).

The LISS panel was started in 2007, and interviews respondents monthly about a wide range of topics. The original sample for the panel was based on a simple random sample of Dutch households, who were contacted and recruited using a mixed-mode design. This design included mail, telephone, and face-to-face as the contact modes. Upon establishing contact with one of the household members, all household members were asked to participate in the panel survey. One member of the household served as a reference person and, as the household informant, provided information about the household. After sample members become panel members, they receive an email invitation on the first Monday of every month with an individualized URL that leads them to the questionnaire of that particular month.

The time required to complete questionnaires in the LISS panel is about half an hour per month, and respondents receive an incentive of $€ 15$ for every hour of completing questionnaires. ${ }^{3}$ They are reminded in case of initial nonresponse in a specific wave, and occasionally receive information about research findings.

New panel members were added to the panel at the end of 2009 and 2011. Most of the new sample members came from top-up samples drawn at those times. In a top-up sample, new respondents are recruited into the panel using a new random sample. In 2009, the top-up sample was stratified based on the inverse response propensities of the original sample, whereas the 2011 top-up sample was again a simple random sample. Other new sample members who entered in 2009 and 2011 consist of household members who have reached the age of 16 and people who have entered the household as a new partner of a panel member.

As long as respondents complete at least one questionnaire item in a particular month, respondents in our study are considered to have participated in that wave. The binary response/nonresponse data from the first 48 waves of the LISS panel serve as our primary dependent variable for studying attrition patterns. As covariates, we will use population data from Statistics Netherlands (2012). For some of these statistics, we have validation information about every sample member. In particular, the variables that we use from the population register are urbanicity of the geographical area and household size. This information was supplemented with data from the recruitment interview. During the recruitment interview, respondents were asked about the household composition and whether they were a home-owner. They were also asked about the age and gender of all other members of

\footnotetext{
${ }^{3}$ Time to complete the survey is estimated beforehand, and every panel member is paid the same corresponding amount.
} 
Table 6.1 Panel recruitment and initial nonresponse rates at various stages.

\begin{tabular}{lcc}
\hline Sampled & $\begin{array}{c}\text { Households } \\
\text { N }=9844^{*}\end{array}$ & $\begin{array}{c}\% \text { of sampled } \\
100\end{array}$ \\
\hline Contact successful & 8911 & 91 \\
Completed recruitment interview or central & 7335 & 75 \\
$\quad$ questions & & 53 \\
Willing to become panel member & 5217 & 48 \\
Household registered as panel member & 4722 & \\
\hline
\end{tabular}

*After panel recruitment was completed, 454 households that were recruited into the panel during a pilot study in 2007 were added to the panel. These households are excluded from Table 6.1, because the pilot study was mainly used for experimentation into successful recruitment strategies for the panel. Including the pilot households, the total number of registered households in the panel is 5005. Within those households, there are 9831 eligible individuals. Of those eligible individuals, 8026 members finally became panel respondents. Some months later, however, a further 122 eligible respondents who first did not want to participate, changed their minds, and did become panel members. These 8148 respondents will be used in our further analyses.

their household. Upon becoming a member of the study, respondents completed a profile questionnaire asking them about their background characteristics. From this dataset, we use a variable that asked respondents about their voting behavior in the Dutch parliamentary elections in 2006. The covariates that we use will not fully explain either nonresponse during the recruitment of the panel, or panel attrition. We use these covariates here for the following reason: these are the best statistics available at the population level to allow for the study of nonresponse and attrition.

\subsubsection{Initial nonresponse}

Because of the availability of the register data from Statistics Netherlands, we are able to study the correlates of initial nonresponse in the recruitment phase of the LISS panel. Initial nonresponse can occur for various reasons (noncontact, refusal to participate at all in the recruitment interview, or refusal to become a panel member, inability to physically or intellectually participate, etc.). For details on the correlates of nonresponse in every stage of the recruitment phase, we refer to Leenheer and Scherpenzeel (2013). Table 6.1 shows that noncontacts amounted to $9 \%$ of all sampled households. At the next stages, more households either refused to participate at all, reported no willingness to become a panel member, or did not complete any online questionnaire. All in all, $48 \%$ of the sampled households registered as panel members. A particular effort was made to offer Internet access to households without Internet access. ${ }^{4}$ This effort was successful, and was especially effective in targeting older respondents (Leenheer \& Scherpenzeel, 2013).

Because panel attrition is an individual attribute, we have chosen to report all our analyses at the individual level. ${ }^{5}$ All the statistics from the recruitment interview as reported in Table 6.2 are therefore weighted for household size, but as a simple random sample was used, no further design weights were used.

\footnotetext{
${ }^{4} 350$ people (4.5\% of panel members) received a computer with Internet connection. Home-Internet coverage in the Netherlands was between $80-90 \%$ in 2008 (Statistics Netherlands, 2008), implying that nonrespondents were more likely than respondents not to have Internet at their home.

${ }^{5}$ In the attrition analyses also the households that were recruited in the pilot experiment are taken into account.
} 
Table 6.2 Sample percentages for panel members, initial nonrespondents, and surviving panel members in the LISS panel.

\begin{tabular}{|c|c|c|c|c|c|c|}
\hline $\begin{array}{l}\text { Sample } \\
\text { percentages }\end{array}$ & $\begin{array}{c}\text { Initial } \\
\text { nonrespondents }\end{array}$ & $\begin{array}{c}\text { Initial panel } \\
\text { members }\end{array}$ & $\begin{array}{l}\text { Active in } \\
\text { April } 2008\end{array}$ & $\begin{array}{c}\text { Active in } \\
\text { April } 2010\end{array}$ & $\begin{array}{l}\text { Active in } \\
\text { Dec } 2011\end{array}$ & Population \\
\hline Over $65 \%$ & $20.3^{*}$ & $10.3^{*}$ & $11.5^{*}$ & $11.2^{*}$ & $11.8^{*}$ & 15.3 \\
\hline \multicolumn{7}{|l|}{$\begin{array}{l}\text { Household } \\
\text { composition }\end{array}$} \\
\hline single $\mathrm{HH}$ & $16.1 *$ & $12.7 *$ & $13.4^{*}$ & $13.4 *$ & $13.8 *$ & 14.9 \\
\hline $\begin{array}{l}\text { couple without } \\
\text { children }\end{array}$ & $22.3^{*}$ & $31.7 *$ & $34.8^{*}$ & $33.9 *$ & $35.3^{*}$ & 27.0 \\
\hline couple with children & 51.4 & 49.4 & $46.6^{*}$ & $47.1 *$ & $45.3 *$ & 50.4 \\
\hline single with children & $7.8^{*}$ & $5.6^{*}$ & $4.7 *$ & $4.9^{*}$ & $4.8^{*}$ & 6.7 \\
\hline other & $1.4^{*}$ & $0.6^{*}$ & $0.6^{*}$ & $0.7 *$ & $0.7 *$ & 1.0 \\
\hline Household size (mean) & $1.91 *$ & 2.58 & $2.83 *$ & $2.88 *$ & $2.83 *$ & 2.24 \\
\hline Home-owner & $61.2^{*}$ & $75.6^{*}$ & $76.3^{*}$ & $76.1^{*}$ & $76.2 *$ & 68.4 \\
\hline \multicolumn{7}{|l|}{ Urbanicity } \\
\hline extremely urban & $25.8 *$ & $12.9 *$ & $13.4^{*}$ & $13.2 *$ & $12.5^{*}$ & 19.4 \\
\hline very urban & $20.6^{*}$ & $25.7 *$ & $26.4^{*}$ & $25.9 *$ & $26.4^{*}$ & 23.1 \\
\hline moderately urban & $14.5^{*}$ & $22.2 *$ & $22.1 *$ & $22.7 *$ & $23.0 *$ & 18.3 \\
\hline slightly urban & $15.9 *$ & $23.0 *$ & $22.5^{*}$ & $22.6^{*}$ & $22.3 *$ & 19.4 \\
\hline not urban & $23.4^{*}$ & $16.2^{*}$ & $15.7^{*}$ & $15.7 *$ & $15.9 *$ & 19.8 \\
\hline \multicolumn{7}{|l|}{ Voting behavior 2006} \\
\hline $\begin{array}{l}\text { CDA (Christian } \\
\text { democrats) }\end{array}$ & - & - & 25.2 & 25.3 & 25.5 & 26.5 \\
\hline PvdA (labor) & - & - & $19.4 *$ & $19.4 *$ & 19.9 & 21.2 \\
\hline VVD (liberals) & - & - & 15.7 & 15.4 & 15.3 & 14.7 \\
\hline SP (socialists) & - & - & 17.3 & 17.9 & 17.7 & 16.6 \\
\hline other parties & - & - & $22.4^{*}$ & 21 & 21.6 & 21 \\
\hline $\mathrm{N}$ & $9878^{\mathrm{a}}$ & 8148 & 7627 & 6892 & 6248 & \\
\hline
\end{tabular}

Notes: 1 Dutch population statistics correspond to individually based statistics, with January 1st 2009 as the base date. Statistics can be found on http://statline.cbs.nl (Statistics Netherlands, 2008).

2 April 2008 was chosen as the start date for showing panel composition, because of the fact that prior to this date, some respondents were still being recruited into the panel.

3 Panel members are respondents who participated in at least one interview until April 2008. We considered a respondent to be an "active" member at any point in time when he/she completed a survey in that particular month, or any of the two preceding months.

4 Household statistics (in the recruitment phase) represent statistics based on individual household members.

Source: Eurostat http://epp.eurostat.ec.europa.eu/statistics_explained/index.php/Housing_statistics

$5 *$ : significant difference with the population using a Chi-square test with $\alpha=.01$. For the population, a sample size equal to the number of panel members was used in conducting the Chi-square test.

6 Statistics in bold: significant difference with initial panel members with $\alpha=.01$. Independent sample Chi-square tests were run for the difference between panel members and nonrespondents, and paired sample t-tests for proportions for the difference between panel members and active panel members in a particular wave.

${ }^{\text {a }}$ This number is computed as the nonrespondents from nonresponding households plus nonrespondents from responding households. The number of nonresponding households (5122 - see Table 6.1) is multiplied by the average household size for nonresponding households minus the number of children living in under 16 (who are ineligible) to estimate the number of eligible individuals in nonresponding households. This amounts to $5122 *(1.91-0.31)=8195$ respondents.

The number of nonrespondents in responding households is computed by taking the difference between the panel members and eligible respondents from all responding households $(9831-8148=1683-$ see Table 6.1$)$. In total, the number of nonrespondents thus amounts to about $8195+1683=9878$. Note that this excludes nonresponse at the household level during the pilot phase of the LISS panel. 
Columns 2 and 3 of Table 6.2 show the percentage of sample members who remained nonrespondents in the panel recruitment phase (column 2) and those who were successfully recruited into the panel (column 3). Because of the fact that a simple random sample of households was drawn, any differences that we find between recruited panel respondents and the general Dutch population, are caused by nonresponse (including noncontact and noncoverage). We find that panel members: (1) are less likely to be older (over 65 years old); (2) are less likely to come from single households; (3) are less likely to come from extremely urban areas; and (4) are more likely to be home-owners than are the nonrespondents. When compared to the population statistics, shown in the last column, it is clear that especially older respondents, respondents from extremely urban areas and single households are missing disproportionally from the panel members at the start of the LISS panel.

In columns 4-6, the same statistics are shown at various stages of the LISS panel for all active panel members at that moment. We see that, although slowly, the initial bias that we find for older respondents is decreasing, meaning that older respondents, when they do become a panel member, are less likely to attrite. A similar trend is found for single households, but biases get larger over time for household size and urbanicity and the proportion of singles living with children.

Apart from focusing on socio-demographic characteristics, we also show statistics for one of the substantive variables in the panel for which we have validation data: voting behavior in the Dutch parliamentary elections of 2006. Online panels often are used for opinion polls, and voting behavior is a common topic in such polls. ${ }^{6}$ We see that for voting behavior, minor biases in the LISS panel do exist. The proportion of voters for the Christian Democrats and Labor Party is underestimated, while it is overestimated for the Liberals and Socialists. Differences with the population, however, are statistically insignificant.

From Table 6.2, we see that nonresponse and attrition biases in the LISS panel vary over time. This implies that attrition is differential among respondents, or stated differently, that specific groups of respondents are less likely to drop out than others. Furthermore, we find that developments in biases are not consistent. For example, the bias in the proportion of panel members who are over 65 years of age, does not become consistently better or worse over time. Obviously, an approach that studies attrition patterns in more detail will help to illuminate this process further.

\subsection{Attrition modeling and results}

To model the binary response data for all 48 waves in our study, we have used a Latent Class model. The advantage of using Latent Classes over other methods is that respondents are categorized on the similarity of their response patterns. This does not mean that all respondents within one latent class follow the exact same response pattern. If our data show classes that either always or never participate, this will mean that all respondents who have a high propensity to be a member of that class will closely resemble these extreme types. However, there will also be classes that vary more in their response data. In fact, because every respondent will be assigned to one or more classes, it is likely that some classes still resemble heterogeneous response patterns.

\footnotetext{
${ }^{6}$ Because voting behavior is an individual attribute, we cannot show statistics for the recruitment phase of the LISS panel, as these statistics are all based on household reports.
} 
We treat a response (i.e., completing at least one item in the questionnaire) to a particular wave as 1 , and non-response as 0 . We did not have a clear hypothesis regarding the number of classes we expected to find, but it is likely that at least 1 class of attriters, 1 class of staying (i.e., non-attriting), and 2 classes that reflect the refreshment samples in 2009 and 2011 will result from our analyses. In addition to this, we believed it was likely that we would find some additional classes describing different patterns of attrition. The attrition pattern within the Latent Classes can take any form; the model is estimated without any constraints on the attrition patterns, and because of that, the solution is entirely based on grouping respondents with similar attrition patterns.

Principally, the fit of our Latent Class model will improve with any additional class that we add to our model, possibly leading to a situation where we have as many latent classes as respondents. To overcome this problem, we used the Bayesian Information Criterion (BIC) as our heuristic for comparing the quality of models with different numbers of classes. Lower values for BIC indicate a better relative fit of the model to the data. As absolute differences for BIC between competing models can be small, it is desirable to test whether any difference between two models is also significant. A Bootstrapped Likelihood Ratio Test is in this case advisable, but we opted for the Lo-Mendell Rubin test, because of the fact that we simultaneously corrected our standard errors for the fact that individual respondents were clustered in households (Nylund, Asparouhov, \& Muthén, 2007). Apart from focusing on the fit values as indicated by the BIC, we also inspected values of the entropy (Celeux \& Soromenho, 1996) as a criterion for the classification quality. The entropy lies between 0 and 1 , where 1 means that every individual can be assigned to a particular class without any measurement error, and 0 means that respondents are randomly assigned to a latent class. Entropy values above .80 are considered to be good (Celeux \& Soromenho, 1996).

Finally, we wanted to avoid substantive solutions that included classes with fewer than $5 \%$ of all respondents, reflecting a minimum of about 500 respondents per class. This lower limit is somewhat arbitrary, but it is likely that very small classes present only a variant on a pattern that is found in one of the larger classes. All models are estimated using MPLUS 6.12 (Muthén \& Muthén, 2011).

Table 6.3 shows the fit statistics of all models with 1-12 classes we tested. From Table 6.3, we prefer the model with nine Latent Classes. The solutions with 10, 11 and 12 classes, respectively, do fit our data better when looking at the BIC. The smallest class of respondents in those solutions, however, includes fewer than 500 respondents. Closer inspection of the attrition pattern for the smallest classes in the solutions with 10-12 classes showed that these classes followed an attrition pattern that mimicked the attrition pattern of the group of slow attriters, though at a somewhat faster or slower rate. For this reason, we chose the model with nine classes as our final model.

Figure 6.1 shows the results of the model with nine latent classes. For each class the response propensities shown indicate the proportion of respondents within each class that participated in each wave of the panel study. ${ }^{7}$ The most loyal respondents together (Class 1 -stayers) comprise about $20 \%$ of the total sample. These respondents participatedin almost all waves of the panel, and showed response propensities close to 1 .

\footnotetext{
${ }^{7}$ From Figure 6.1, one can distill that drop-out occurred disproportionally in waves 8 and, to a lesser extent wave 22 of the study. We believe this temporary drop-out to be caused by the topic of those months' questionnaires. The questionnaire of wave 8 was introduced in the invitation as a complex questionnaire on each household's income and assets. In most of attriting classes, respondents returned after this wave, but for respondents who were already in the process of dropping out, receiving this questionnaire only quickened the attrition process.
} 
Table 6.3 Model fit, entropy and smallest class sizes of the Latent Class models for attrition.

\begin{tabular}{llccc}
\hline & BIC & $\begin{array}{c}\text { Lo-Mendell Rubin } \\
\text { test } p \text {-value }\end{array}$ & Entropy & Min. class size \\
\hline 1-class & 836566 & - & 1 & 12476 \\
2-class & 527401 & $p<0.01$ & .991 & 6057 \\
3-class & 449638 & $p<0.01$ & .992 & 1580 \\
4-class & 393778 & $p<0.01$ & .987 & 1565 \\
5-class & 372274 & $p<0.01$ & .978 & 1484 \\
6-class & 359605 & $p<0.01$ & .974 & 1126 \\
7-class & 349077 & $p<0.01$ & .974 & 874 \\
8-class & 343771 & $p=0.11$ & .972 & 621 \\
9-class & $\mathbf{3 3 8 6 7 4}$ & $p<0.01$ & .967 & $\mathbf{5 7 3}$ \\
10-class & 335601 & $p<0.01$ & .966 & 438 \\
11-class & 332204 & $p=0.01$ & .965 & 318 \\
12-class & 329224 & $p<0.01$ & .964 & 314 \\
\hline
\end{tabular}

Note: The Lo-Mendell Rubin tests whether the class solution with 1 class less, fits the data significantly worse than the model with the current class. The class shown in bold is the preferred model.

A second group (Class 2 - Slow Attriters), comprising 13\% of the panel, has a stable response propensity at a slightly lower rate, between 0.8 and 0.9 . Towards the end of the period, their response propensities do decline to about 0.6.

Three groups follow a traditional pattern of attrition. The fastest group of this subset (Class 6 - Fast Attriters), about $12 \%$ of the sample, starts to drop out from the first wave of the panel study, and essentially have dropped out altogether after one year. A second group of this subset (Class 5; 8\%) starts to attrite after 1 year. The final group of this subset attrites after about two years of panel membership (Class 3; $4 \%$ of the sample).

In addition, we see a group that participates infrequently throughout the panel, showing consistent response propensities between 0.4 and 0.6 , indicating that they participate in about every second wave of the panel survey. We call this group "lurkers" (Class 4).

The three remaining classes consist of respondents who were added to the sample over the course of the panel. Two classes are formed by the group of respondents entering the panel in 2009 (the largest one staying [Class 8], but a smaller one dropping out [Class 9]). The final class (Class 7) consists of respondents who, though consenting to become a panel member during the recruitment interview, never start participating. The increase in response propensities in the last two waves for this class is caused by the fact that this also includes a group of top-up respondents and children reaching the age of 16 at the end of 2011, who both enter the panel in the final two waves of our study.

The value for the entropy in the model with 9 classes is very high (.967), implying that there is very little classification error. This means that almost every respondent can accurately be assigned to be a member of one specific class. From here on, we use the Most Likely Class membership to categorize every respondent and look at the composition of each attrition class for the solution with nine Latent Classes.

Table 6.4 shows the composition of each of the classes on socio-demographic background variables. We focus our analyses here on the original sample members who were recruited into the panel in 2007 and leave the top-up sample members in 2009 and 2011 out of the analyses. 


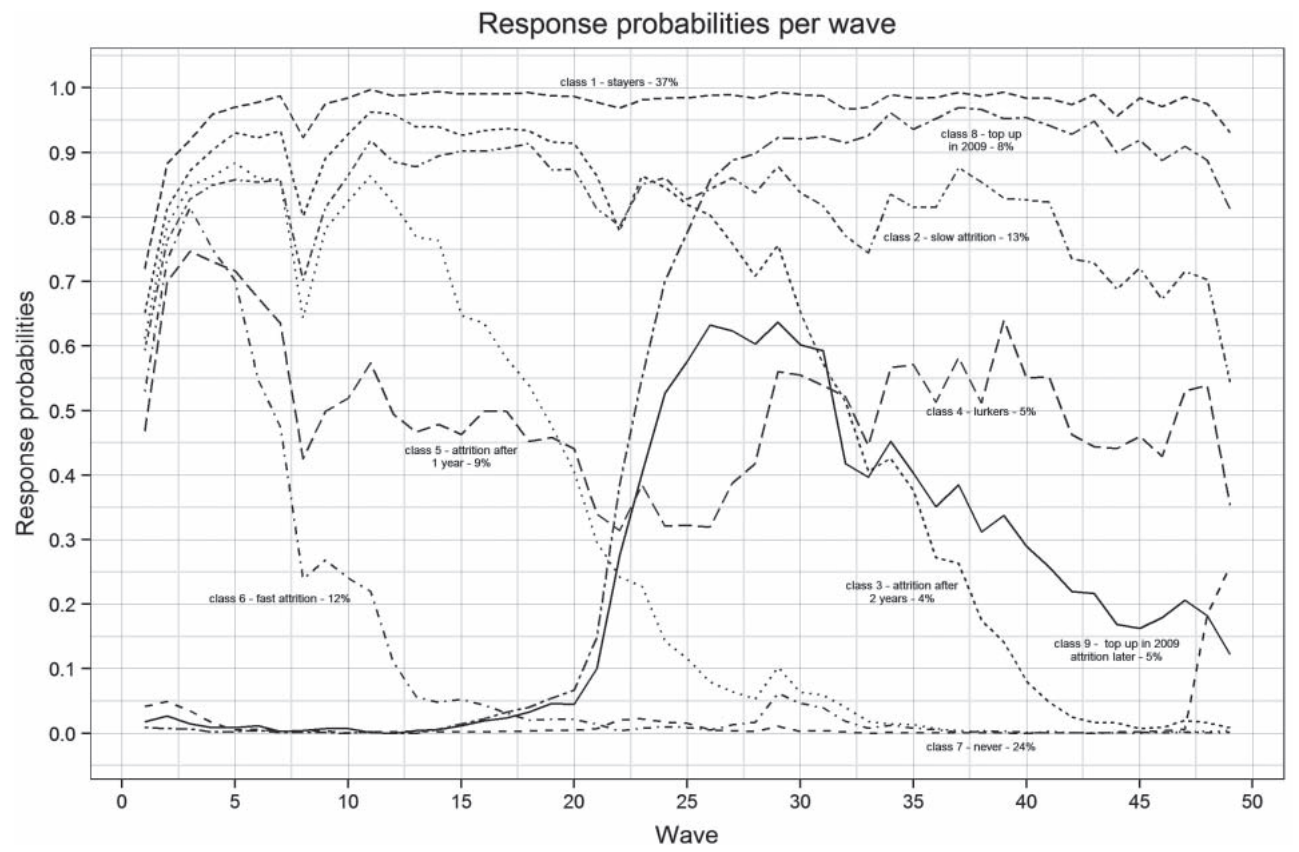

Figure 6.1 Attrition patterns for the nine Latent Classes and their sample fractions. 


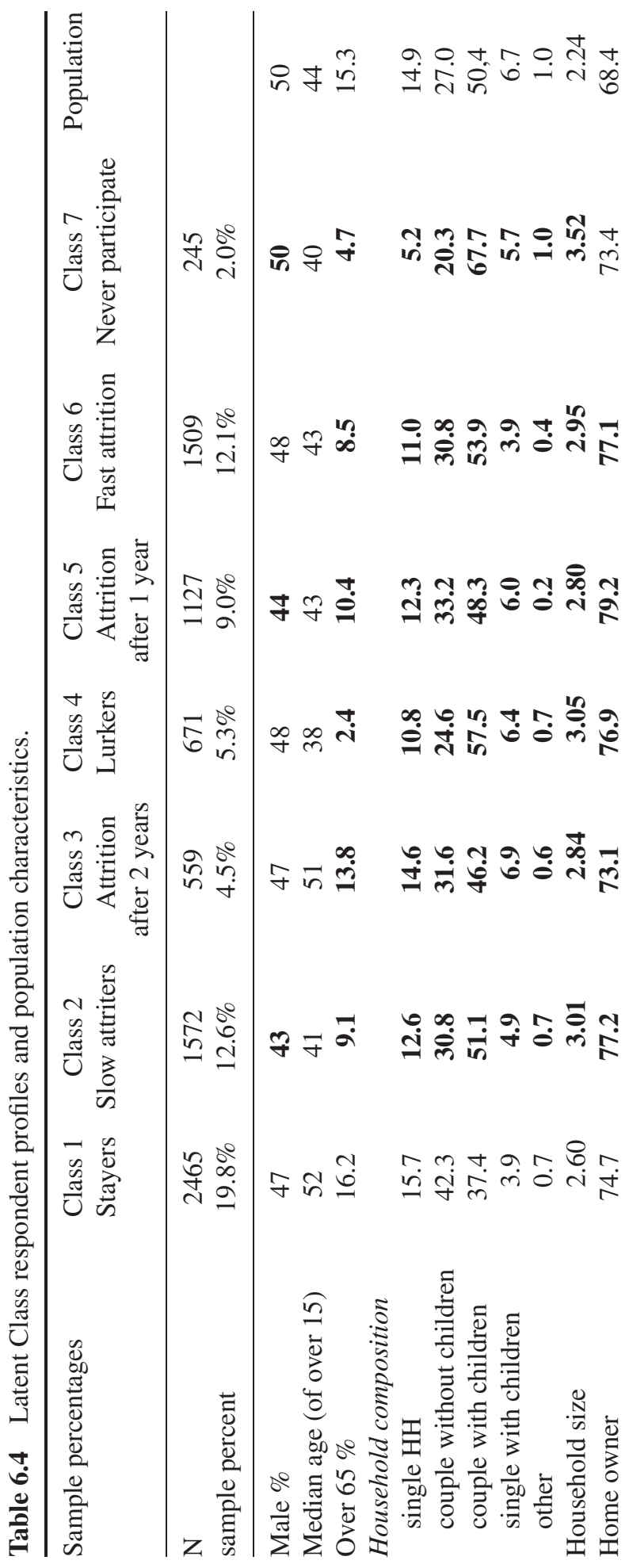




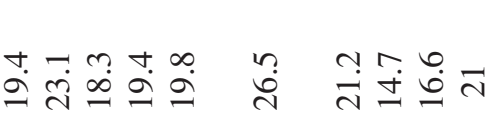

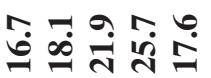

ํํำ

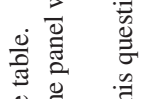

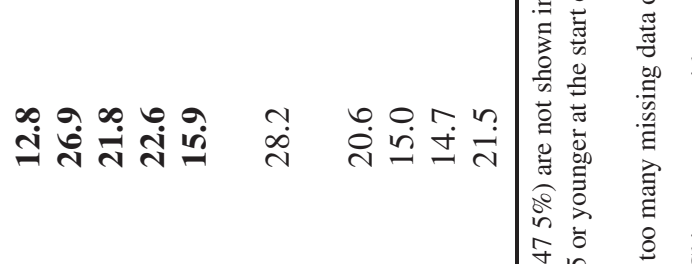

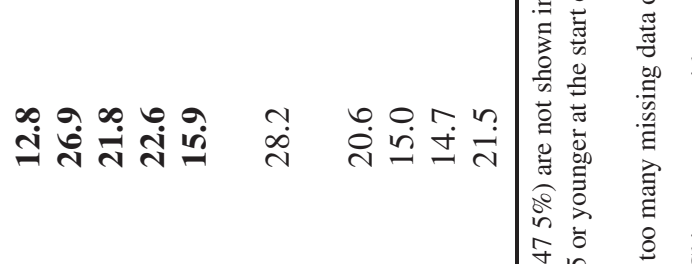

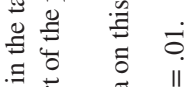

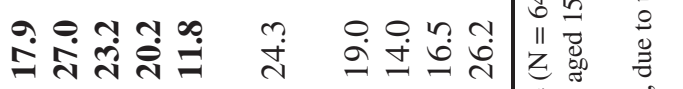

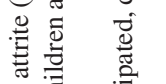

ஸ்

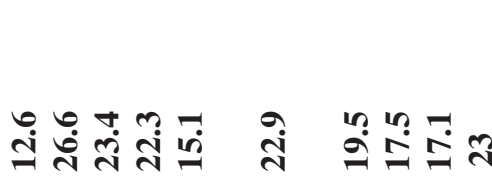

m n m n a ?

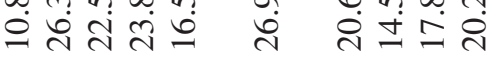

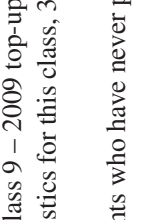

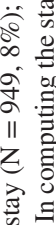

㺃

言

它远

令苟

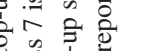

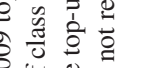

ते

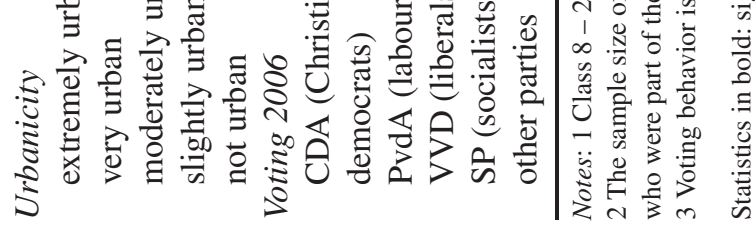


Also, we use the statistics as recorded at the start of the panel, and do not account for any changes in, for example, household composition that occurred over the course of the panel. Household composition and household size do of course change for respondents, but accounting for such changes would make it necessary to work with time-variant covariates at each of our 48 measurement occasions, making model estimation unacceptably time-consuming.

As shown in Table 6.4, large differences exist between the classes. Among our initial sample members, the stayers (Class 1) form the first class in our data (20\%). These respondents are more likely to be older, come from single households or live with someone else as a couple without children, and are less likely to live in extremely urban areas than respondents in all other classes.

The class that is most different from the stayers is not surprisingly the group who never participates in the LISS panel. Whereas the median age is highest in the group of stayers, the median age is one of the lowest in the class who never participates (Class 7). People who never participate are least likely to come from single households or be from a couple without children. It is also remarkable that females are overrepresented in all classes, except the class that never participates.

Respondents in all classes differ from each other, but not as much as the stayers differ from respondents who never participate. Those who attrite after two years of panel membership (Class 3) look most like the stayers in terms of age, gender, and household composition. The group of Lurkers (Class 4) resembles the class who never participates quite closely, especially on age and household composition. The other attriting classes sometimes resemble the class of stayers and sometimes the class who never participates; their profile is less clear.

When it comes to differences between the social-demographic composition of every attrition class and the general population, there is no single class that stands out as performing best. All classes seem to differ from the population, but in various ways. Because of attrition, biases in some variables (urbanicity, gender) become worse over time, because the profiles of the attriting classes here come closest to the population estimate. For some other variables (age, household size), biases decrease over time, because for those variables, it is the class of stayers that resembles the population estimate best. The consequences of attrition for estimates of voting behavior are relatively small. Voting percentages for the Christian Democrats were somewhat underestimated at the start of the panel (see Table 6.2), but in the class of stayers, the Christian Democrat vote is well estimated. The only class of respondents that is very different from the population on voting behavior is the class of Fast Attriters. In this class, the percentage of voters for the Christian Democrats and Labor is strongly underestimated.

In conclusion, we find that there are large differences between the different attriting classes. The class of stayers includes respondents who always participate in every wave. We find that every attrition class differs on one or more variables from this class, meaning that attrition itself is selective. When we compare every attriting class to the general population, we see that no class of respondents resembles the population well. In order to answer our last research question, whether initial nonresponse and attrition can be explained by the same process, we take a closer look at the profiles of different groups of nonrespondents in the next section.

\subsection{Comparison of attrition and nonresponse bias}

Table 6.5 combines information on various types of nonrespondents from Tables 6.2 and 6.4. The second column shows the sample statistics for initial nonrespondents in the recruitment 


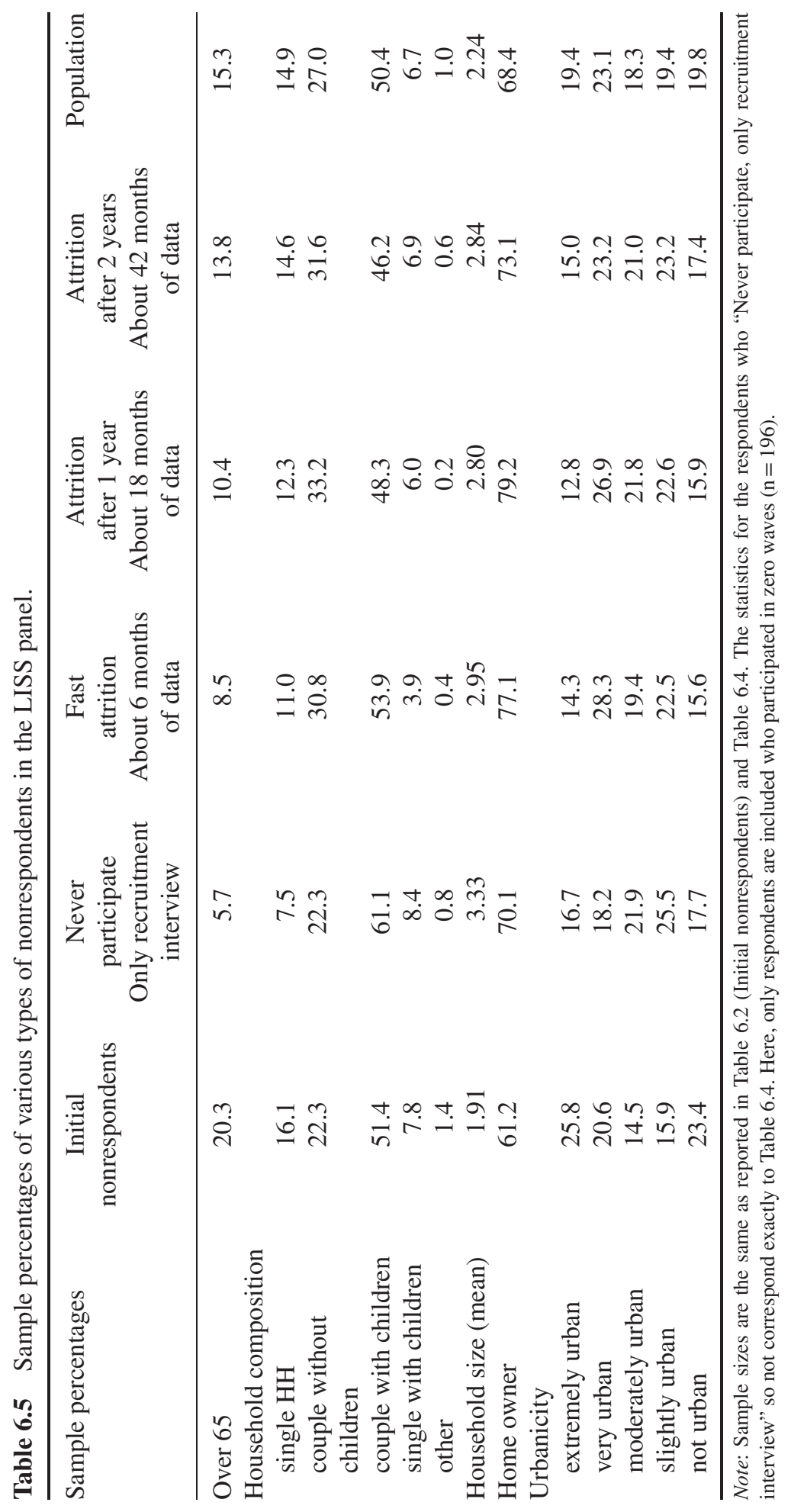


phase of the LISS panel, while the third to the sixth columns show statistics for four Latent Classes of attriters. There are strong differences between nonrespondents in the panel recruitment phase and the class of respondents who never participate. Although both groups of respondents effectively do not participate in the LISS panel, they differ strongly on age, household composition, household size, and urbanicity. People over 65 years old are commonly found among nonrespondents in the recruitment phase of the panel, but hardly among respondents who did consent to participate, but then never do in practice. This might be related to the fact that about $14 \%$ of respondents over 65 years of age received Internet upon consenting to become a panel member; hence quick drop-out among them is unlikely.

All other attrition classes differ from initial nonrespondents as well. One would expect differences between Late Attriters and initial nonrespondents to be larger than for the class that never participates, but we did not find this. The later attrition occurs, the more the class resembles the initial nonrespondents. But even for the most recent attrition class, the differences with initial nonrespondents remain large. In short, nonrespondents in the recruitment phase are very different from all people who attrite from the panel.

\subsection{Discussion and conclusion}

Because of the lack of a sampling frame for sampling the general public via the Internet, several research organizations have established online panels that rely on traditional probability samples. They recruit people using offline survey methods, hoping to overcome the problem of the low external validity of self-selected panels.

This chapter showed how nonresponse and attrition in online panels differ from nonresponse and attrition in offline panels. In general, respondents who cannot use the Internet, or do not like it, are likely to refuse during the recruitment phase to participate in online panels, making initial nonresponse highly selective (nonrandom). The LISS panel that we used in this study addressed this issue by providing households that could not otherwise participate with a computer and an Internet connection. However, we found that initial nonresponse bias was not entirely eliminated, especially for age.

Differences in attrition between online and offline panels are related to the mode of contact. Email invitations are easily ignored or forgotten, leading to higher levels of nonresponse. On top of this, the high frequency of data collection requests that online panel surveys use is also likely to lead to higher wave-nonresponse at any given wave. We used a Latent Class model to study the diffuse patterns of attrition that are the results of this. We found that attrition does not occur gradually or in a linear way. For example, we find a group of "lurkers", i.e., respondents who participate only in about every second wave of the survey, and do so for the entire period of the panel. We also find differences among attrition patterns. There is a group of respondents that drops out very fast, but also a group that does so very slowly, and a group that stays very loyal for the first two years of data collection, then dropping out after this. We further find that substantively all these classes differ from each other. Finally, the correlates of nonresponse in the panel recruitment phase and attrition are very different. This implies that nonresponse during recruitment and panel attrition are different processes. We would welcome studies that try to replicate our findings in other online panel surveys, though such specific characteristics as the frequency of data collection will of course influence the number and size of Latent Classes in every panel survey. This includes replication of our study in a self-selected panel survey. We would suspect that fast attrition does not occur as often in self-selected panels as in our study because of the internal motivation that is necessary to join a self-selected panel, 
but it would, for example, be interesting to see whether lurkers are present to the same extent in self-selected panels as in our analyses.

The LISS panel does not include sample weights that correct for nonresponse and attrition, and our analyses show why providing them is complicated. The number of separate weights that need to be calculated with 48 waves of data is extraordinarily high. Cross-sectional weights could practically still be computed, but computing and using longitudinal weights becomes complicated when users do not use all waves of the LISS in substantive analyses. One solution to this problem would be to let users calculate nonresponse weights using an interactive (online) module. Multiple imputation of missing data, however, seems a more attractive solution to correct for nonresponse and attrition, because it is more readily available in the major software packages, and can deal with item- and unit nonresponse simultaneously. Missing values because of either item- or wave nonresponse can be imputed using all data that were collected in the same and earlier waves of data collection. Another approach that the LISS panel is undertaking is to try to re-activate specific classes of attriters using a tailored design approach. For example, the lurkers may be activated by contacting them more frequently to keep them involved in the panel. Attriting respondents can perhaps be targeted more effectively by offering an extra incentive, or addressing very specific concerns that led the respondents to drop out.

Although this chapter has only discussed attrition and nonresponse in a probability-based online panel, nonresponse and attrition of course exist in self-selected panels as well. Drawing quota samples, as is often done in self-selected panels (see Chapter 8) does not solve the problem of nonresponse, but rather transforms it into a coverage problem. Self-selected panel managers may stratify their quota samples on past response behavior, effectively only sampling from loyal panel members to overcome the problem of attrition. Response rates calculated for self-selected panels therefore are not informative, and hide the problem of nonresponse that also exists in self-selected panels. Our analyses showed, however, that the profile of loyal panel members is very different from that of the general population, and that those respondents who drop out form important strata of the general population. In self-selected panels, such biases will therefore not appear as much as nonresponse bias, but rather as coverage bias during the self-selection process.

Within the framework of probability-based surveys, coverage and nonresponse biases can be assessed, and corrected by inviting (stratified) top-up samples into the study, or using weighting or imputations. Although this may at times be complicated given the complex data structure of probability-based panel surveys, correcting for undercoverage and nonresponse in self-selected panels is impossible. Due to the absence of a sampling frame and frame variables one cannot accurately weight data from the self-selected sample back to the target population. Even in the case when external validation data at the level of the target population are available for a self-selected panel survey, weighting will only reduce biases when the external validation variables adequately explain both the self-selection process and the variable of interest. In self-selected panels, model-based weighting procedures seldom achieve these dual goals, and for that reason one does not know whether any weighting procedure effectively corrects for coverage and nonresponse bias, or only makes it worse.

\section{References}

Behr, A., Bellgardt, E., \& Rendtel, U. (2005). Extent and determinants of panel attrition in the European Community Household Panel. European Sociological Review, 21, 489-512. 
Borsboom, D., Mellenbergh, G.J., \& van Heerden, J. (2004). The concept of validity. Psychological Review, 111, 1061-1071

Campbell, D. T., \& Stanley, J.C. (1966). Experimental and quasi-experimental designs for research. Skokie, IL: Rand McNally.

Celeux, G., \& Soromenho, G. (1996). An entropy criterion for assessing the number of clusters in a mixture model. Journal of Classification, 13, 195-212.

De Leeuw, E. D., \& Hox, J. (2011). Internet surveys as part of a mixed mode design. In M. Das, P. Ester, \& L. Kaczmirek (Eds.), Social and behavorial research and the Internet: advances in applied methods and new research strategies. (pp. 45-76). New York: Routledge.

Durrant, G. B., \& Goldstein, H. (2010). Analysing the probability of attrition in a longitudinal survey. University of Southampton Working Paper, (M10/08).

Fitzgerald, J., Gottschalk, P., \& Moffitt, R. (1998). An analysis of sample attrition in panel data: The Michigan panel study of income dynamics. Journal of Human Resources, 33, 251-299.

Groves, R. M., \& Couper, M. P. (1998). Nonresponse in household survey interviews. New York: John Wiley \& Sons, Inc.

Kroh, M. (2011) Documentation of sample sizes and panel attrition in the German Socio Economic Panel (1984 until 2010). DIW paper 2011-59. Retrieved February 5, 2013, from: http://www.diw.de/documents/publikationen/73/diw_01.c.385005.de/diw_datadoc_2011-059.pdf.

Laurie, H., \& Lynn, P. (2009). The use of respondent incentives on longitudinal surveys. In: P. Lynn (Ed.), Methodology of longitudinal surveys (pp. 205-234). Chichester: John Wiley \& Sons, Ltd.

Leenheer, J., \& Scherpenzeel, A. (2013). Does it pay off to include non-Internet household in an Internet panel? International Journal of Internet Science, 8. Retrieved July 2, 2013 from: http://www.ijis.net /ijis8_1/ijis8_1_leenheer_and_scherpenzeel_pre.html.

Lepkowski, J. M., \& Couper, M. P. (2002). Nonresponse in the second wave of Longitudinal Household Surveys. In R. M. Groves, et al. (Eds.), Survey nonresponse. New York: John Wiley \& Sons, Inc.

Lillard, L. A., \& Panis, W.A. (1998). Panel attrition from the Panel Study of Income Dynamics: Household income, marital status and mortality. The Journal of Human Resources, 33, 437-457.

Lipps, O. (2009). Attrition of households and individuals in panel surveys. SOEP Papers, (164). Retrieved June 28, 2013, from: www.diw.de/documents/publikationen/73/diw_01.c.96125.de/diw _sp0164.pdf.

Lynn, P. (2009). Methods for longitudinal surveys. In P. Lynn (Ed.), Methodology of longitudinal surveys (pp. 1-20). Chichester: John Wiley \& Sons, Ltd.

Muthén, L. K., \& Muthén, B. (2011). MPLUS. Los Angeles: Statmodel.

Nicoletti, C., \& Peracchi, F. (2005). Survey response and survey characteristics: Microlevel evidence from the European Community Household Panel. Journal of the Royal Statistical Society Series A, $168,763-781$.

Nylund, K. L., Asparouhov, T., \& Muthén, B. (2007). Deciding on the number of classes in latent class analysis and growth mixture modeling: A Monte Carlo simulation study. Structural Equation Modeling, 14, 535-569.

Olson, K., Smyth, J. D., \& Wood, H. M. (2012). Does giving people their preferred survey mode actually increase participation rates? An experimental examination. Public Opinion Quarterly, 76, 611-635.

Schaan, B. (2011). Fieldwork monitoring in SHARELIFE. In M. Schröder (Ed.), Retrospective data collection in the survey of health ageing and retirement in Europe (pp. 29-36). Mannheim: SHARELIFE Methodology, MEA.

Scherpenzeel, A., \& Das, M. (2011). True longitudinal and probability-based internet panels: Evidence from the Netherlands. In M. Das, P. Ester, \& L. Kaczmirek (Eds.), Social and behavorial research and the internet: Advances in applied methods and research strategies (pp. 77-104). New York: Routledge. 
Sikkel, D., \& Hoogendoorn, A. (2008). Panel surveys. In E. D. de Leeuw, J. J. Hox, \& D. A. Dillman (Eds.), International handbook of survey methodology (pp. 479-499). New York: Lawrence Erlbaum.

Statistics Netherlands (2008). No Internet access for 1.2 million Dutch people. web magazine 18 December 2008. Retrieved June 14, 2013, from: www.cbs.nl/en-GB/menu/themas/vrije-tijd -cultuur/publicaties/artikelen/archief/2008/2008-2641-wm.htm.

Statistics Netherlands (2012). Statline database. Retrieved February 4, 2013, from: http://statline.cbs.nl /statweb/?LA=en.

Tortora, R. D. (2009). Attrition in consumer panels. In P. Lynn (Ed.), Methodology of longitudinal surveys (pp. 235-248). Chichester: John Wiley \& Sons, Ltd.

Uhrig, S. N. (2008). The nature and causes of attrition in the British household panel survey. ISER Working Paper (5). Retrieved June 28, 2013, from: https://www.iser.essex.ac.uk/publications /working-papers/iser/2008-05.

Voorpostel, M. (2009). Attrition in the Swiss household panel by demographic characteristics and levels of social involvement. FORS Working Paper, 1_09. Retrieved June 28, 2013, from: http://aresoas.unil.ch/workingpapers/WP1_09.pdf.

Voorpostel, M., \& Lipps, O. (2011). Attrition in the Swiss household panel: Is change associated with drop-out? Journal of Official Statistics, 27, 301-318.

Watson, N., \& Wooden, M. (2009). Identifying factors affecting longitudinal survey response. In P. Lynn (Ed.), Methodology of longitudinal surveys (pp. 157-182). Chichester: John Wiley \& Sons. Ltd. 